\title{
Structural, Dielectric, and Interface Properties of Crystalline Barium Silicate Films on Si(100): A Robust High- $\kappa$ Material
}

\author{
S. Islam, ${ }^{1}$ K. R. Hofmann, ${ }^{2}$ A. Feldhoff, ${ }^{3}$ and H. Pfnür ${ }^{1, *}$ \\ ${ }^{1}$ Institut für Festkörperphysik, ATMOS, Leibniz, Universität Hannover, Appelstraße 2, \\ D-30167 Hannover, Germany \\ ${ }^{2}$ Institut für Materialien und Bauelemente der Elektronik, Leibniz Universität Hannover, \\ Schneiderberg 32, D-30167 Hannover, Germany \\ ${ }^{3}$ Institut für Physikalische Chemie, Leibniz Universität Hannover, Callinstraße 1, \\ D-30167 Hannover, Germany
}

(Received 7 December 2015; revised manuscript received 29 February 2016; published 9 May 2016)

\begin{abstract}
The quality and crystallinity of ultrathin dielectric layers depend crucially on the details of interface formation and chemical stability. Using a combination of photoelectron (XPS) and electron-energy-loss spectroscopy, low-energy electron-diffraction, and transmission electron microscopy (TEM), we show that crystalline epitaxial layers of $\mathrm{Ba}_{2} \mathrm{SiO}_{4}$ can be grown on $\mathrm{Si}(100)$ substrates from evaporated $\mathrm{Ba}$ in oxygen background atmosphere at $650^{\circ} \mathrm{C}$. Since the silicate is chemically by far more stable than the oxides of $\mathrm{Si}$ and $\mathrm{Ba}$, an atomically sharp interface with no interface oxide is formed, as confirmed by XPS and TEM. However, the interface is rough on the atomic scale. dc and frequency-dependent electrical measurements reveal a relative dielectric constant of 22.8 , low hysteresis in $C V$ measurements, and low leakage currents but still fairly high interface trap densities.
\end{abstract}

DOI: 10.1103/PhysRevApplied.5.054006

\section{INTRODUCTION}

The CMOS transistor based on silicon is still by far the most important switching element in electronic circuits. The progressive miniaturization following Moore's law is only possible by implementation of alternative gate oxides with higher dielectric constants $\epsilon_{r}$ than $\mathrm{SiO}_{2}$. These alternative gate oxides have to fulfill a number of specific physical and technological requirements regarding $\epsilon_{r}$, thermodynamic and kinetic stability, interface and bulk defects [1], and several others. Although the focus of application and research in recent years has been on $\mathrm{HfO}_{2}$ [2], there is still an intense search for other alternatives to $\mathrm{SiO}_{2}$ that avoid the nonidealities of $\mathrm{HfO}_{2}[1,2]$, in particular, the tendency to form $\mathrm{SiO}_{2}$ at the interface.

Our study of ultrathin crystalline Ba-silicate layers on $\mathrm{Si}(100)$ is partly motivated by this context, presenting a route to a gate dielectric that guarantees the absence of any traces of $\mathrm{SiO}_{2}$ at the interface for thermodynamic reasons, in contrast to other silicates that have been considered for the use as high- $\kappa$ materials such as the silicates of $\mathrm{Y}[3,4], \mathrm{Hf}, \mathrm{Zr}$, or La [5-7]. These materials typically exhibit a miscibility gap so that they tend to decompose into the respective oxides, which results in a comparatively low $\epsilon_{r}$ [1].

On the other hand, the system investigated here represents an interesting study object of epitaxy, interface physics, and interface chemistry, as well as of electronic transport along and through semiconductor-insulator

*pfnuer@fkp.uni-hannover.de interfaces. The interfaces between insulating ultrathin films provide a wealth of new physical properties compared with the individual single components with many aspects of functionalization and applications. While in tunnel junctions and field-effect transistors in strongly correlated electron systems [8,9], e.g., the interface is responsible for band alignment and, thus, for the effective barrier height, interface scattering is important for the efficiency of spin filters yielding large tunneling magnetoresistances [10]. On the other hand, magnetic ordering happens at the interface of materials that are nonmagnetic in the bulk [11]. Because of strong polarization and charge transfer, such interfaces can even be conducting [12] and superconducting [13]. This shows that ultrathin films and the interfaces of these materials are of ever-increasing interest.

The interface between silicon surfaces and dielectric layers has been investigated for a long time but still is of high scientific and, of course, also of technological interest for constructing field-effect transistors. The search for most suitable gate dielectric materials has been concentrating on oxides over the last 20 years [1,14-20]. Also, ternary compounds such as titanates or aluminates have been discussed (see, e.g., Refs. [21-25]). The mechanisms of chemical bond formation between the semiconductor and the dielectric material and their stability determine important quantities like the sharpness of the interface, trap densities, or band alignment [16]. Indeed, there is an intriguing interplay between chemical, structural, and kinetic properties at the interface that determine the growth modes of the dielectric layers [26,27]. 
High chemical reactivity and insufficient thermal stability are general problems. As an example, epitaxial films of mixed $\mathrm{Ba}$ and $\mathrm{Sr}$ oxide layers on $\mathrm{Si}$, which we investigated previously [28-33], were shown to grow with perfect lattice match to $\mathrm{Si}(100)$. They grow in crystalline form only after introduction of an intermediate Sr layer [28], thus, demonstrating again the importance of interface formation [27]. The importance of details of the interface is also demonstrated by the large difference in interface trap densities between amorphous and crystalline oxides. Whereas for the crystalline material, the interface trap density at midgap is found to be as low as $6 \times 10^{10} \mathrm{~cm}^{-2} \mathrm{eV}^{-1}$, the amorphous oxide grown without $\mathrm{Sr}$ interface layer has values above $10^{12} \mathrm{~cm}^{-2} \mathrm{eV}^{-1}$ [30]. The excellent electrical properties contrast with its limited thermal stability. By thermal treatment in vacuo at temperatures above $400^{\circ} \mathrm{C}$, it irreversibly transforms into silicates [32]. Si turns out to be the most mobile component, so that the chemical reaction takes place by diffusion from the Si bulk into the oxide layer. No $\mathrm{SiO}_{2}$ is formed during silicate formation. This, in fact, is to be expected for thermodynamical reasons [34]: Silicates of alkaline earth metals are chemically significantly more stable than a mixture of the oxides of $\mathrm{Si}$ and of alkaline earth metals. Silicates are, therefore, spontaneously generated at elevated temperatures from the oxides [35,36]. These silicate layers are found to be stable up to a desorption temperature above $750^{\circ} \mathrm{C}$ [32]. The thermal stability is, thus, comparable to $\mathrm{SiO}_{2}$.

In our study presented here, we combine structural investigations of bulk and interface between $\mathrm{Si}$ and $\mathrm{Ba}$ silicate carried out with low-energy electron-diffraction (LEED) and transmission electron microscopy (TEM) with photoelectron spectroscopy (XPS) in order to determine chemical properties and band offsets, and electrical measurements on metal-insulator-semiconductor diodes (frequency-dependent $C V$ and $I-V$ ), which allow determination of $\epsilon_{r}$, bulk and interface trap density, as well as leakage currents. We find that the identification of improved growth conditions to achieve crystalline growth is important for a physical understanding of the electrical properties of ultrathin crystalline Ba-silicate films. As we will show, this class of material fulfills already now many of the requirements mentioned above. In particular, it combines a high value of band gap $(5.7 \mathrm{eV})$ with a large value of the dielectric constant $\epsilon_{r}=22.8$ ) and high thermal stability. Therefore, it may be well suited as an alternative gate dielectric.

\section{EXPERIMENT}

$\mathrm{Ba}_{2} \mathrm{SiO}_{4}$ layer of thicknesses between 2 and $12.5 \mathrm{~nm}$ are prepared under UHV conditions (base pressure $2 \times 10^{-10}$ mbar) on $\mathrm{Si}(100)$ samples (size $15 \times 15 \mathrm{~mm}^{2}$, $p$-type, $\mathrm{B}$ doped with $\mathrm{N}_{A}=3.5 \times 10^{15} \mathrm{~cm}^{-3}$ ). Flat unstructured $\mathrm{Si}$ is cleaned ex situ by oxidation and reduction cycles. In vacuum, it is carefully degassed for many hours at temperatures up to $550{ }^{\circ} \mathrm{C}$, and the oxide film is finally removed by flash annealing to $1150{ }^{\circ} \mathrm{C}$. $\mathrm{Ba}_{2} \mathrm{SiO}_{4}$ films are generated by evaporation of $\mathrm{Ba}$ from a crucible by electron-beam heating in an ambient oxygen pressure of $3 \times 10^{-7}$ mbar, keeping the Si sample at $650^{\circ} \mathrm{C}$. The $\mathrm{Ba}$ flux is controlled by quartz microbalances close to the evaporator and at sample position. The oxygen background pressure is adjusted so that a well-defined chemical species with sharp diffraction spots is generated, as judged by photoelectron spectroscopy (XPS), by spot profile analysis in low-energy electron (SPALEED), and finally verified by TEM. TEM cross-sectional images also serve for final precise calibration of silicate thickness.

Unstructured samples are investigated using XPS, SPALEED, electron-loss spectroscopy (EELS), and TEM, partly at high resolution (HRTEM). The TEM and HRTEM investigations are carried out in a field-emission transmission electron microscope (FE TEM) from JEOL (JEM-2100F), which is also equipped with an energydispersive $\mathrm{x}$-ray spectrometer. TEM images and HRTEM dark-field images are taken in cross-sectional views to observe the silicate structure, its thickness, nature of growth, and crystallinity.

MOS test capacitors are fabricated on 4-in wafers of the same $\mathrm{Si}$ material. The wafer surface is covered with a thermally grown 200 -nm-thick field oxide $\left(\mathrm{SiO}_{2}\right)$, which is subsequently structured by optical lithography and wet etching to obtain active windows of various sizes between $A=6.4 \times 10^{-7} \mathrm{~m}^{2}$ and $4.0 \times 10^{-8} \mathrm{~m}^{2}$. Ohmic back contacts are produced by implantation of Ta $\left(5 \times 10^{15} \mathrm{~cm}^{-2}\right.$, $40 \mathrm{keV}$ ), thermal annealing, and by sputtering of tantalum nitride. After extremely careful chemical removal of the resist in several steps of dilution, the cleaning of the bottom surface within the windows is done by oxidation and reduction cycles. Finally, a hydrogen-terminated Si surface is produced by dipping into HF with $1 \%$ concentration. The sample is then immediately put into UHV with a mask mounted on top for the definition of the electrically separated metal gates covering the windows. After a first heating step to $850^{\circ} \mathrm{C}$, which removes the hydrogen, the silicate is now grown without any wetting layer at elevated temperatures of $650{ }^{\circ} \mathrm{C}$ and with further annealing up to $700^{\circ} \mathrm{C}$ for 30 to $45 \mathrm{~min}$ after deposition. The grown silicate films are covered with a 150-nm-thick Au capping layer evaporated as an overlapping metal gate. This capping layer also serves as a seal for ambient moisture. In order to improve adhesion, we use one monolayer (ML) of $\mathrm{Al}$ between silicate and gate in our MOS diode. This layer turns out to be crucial for the wetting of Au on oxide [33].

\section{RESULTS AND DISCUSSION}

\section{A. Reactive growth}

As a first step, we adjust the flux of Ba relative to the oxygen pressure and optimize it with respect to 
stoichiometry and crystalline order. For this purpose, we compare the XP spectra of the $\mathrm{Ba}_{2} \mathrm{SiO}_{4}$ layers with those obtained earlier [32]. $\mathrm{O} 1 s, \mathrm{Ba} 3 d$, and $\mathrm{Si} 2 p$ signals are measured and optimized for chemical uniformity. Sample spectra of the Si2 $p$ signals are shown in Fig. 1. Compared with the spectrum of the clean $\mathrm{Si}(100)$ surface, the peak appearing at a binding energy of $101.6 \mathrm{eV}$ is attributed to the formation of the silicate. Because of the increasing silicate thickness, the Si peak at $99.2 \mathrm{eV}$ of the underlying $\mathrm{Si}$ sample is damped accordingly. The spectrum obtained with $3 \mathrm{ML} \mathrm{BaO}$ not shows only the damped contribution of the $\mathrm{Si}$ substrate, it also contains a contribution from the bonding between $\mathrm{BaO}$ and $\mathrm{Si}$ through oxygen at $100.4 \mathrm{eV}$, visible here as $\mathrm{Si}-\mathrm{O}$ formation at the interface. This contribution saturates within the first layer, as has been analyzed in detail previously [28]. After transformation into silicate upon annealing at $650{ }^{\circ} \mathrm{C}$, the silicate peak at $101.6 \mathrm{~V}$ appears as the only new peak. This situation is unchanged when the silicate layer is grown directly at a substrate temperature of $650{ }^{\circ} \mathrm{C}$, demonstrating chemical stability of this layer.

We also note again that no traces of silicon dioxide can be detected, as expected, since even for the reaction of $\mathrm{BaO}$ and $\mathrm{SiO}_{2}$ the Gibbs free energy is strongly negative [34].

\section{B. Epitaxy and crystalline properties}

The Ba-silicate layers formed at $650{ }^{\circ} \mathrm{C}$ are then investigated with LEED and with TEM. We first concentrate on the LEED results, which are shown in Fig. 2. Here we show as an example a 2.5 -nm-thick Ba-silicate film, together with a schematic of the LEED image for better orientation. The

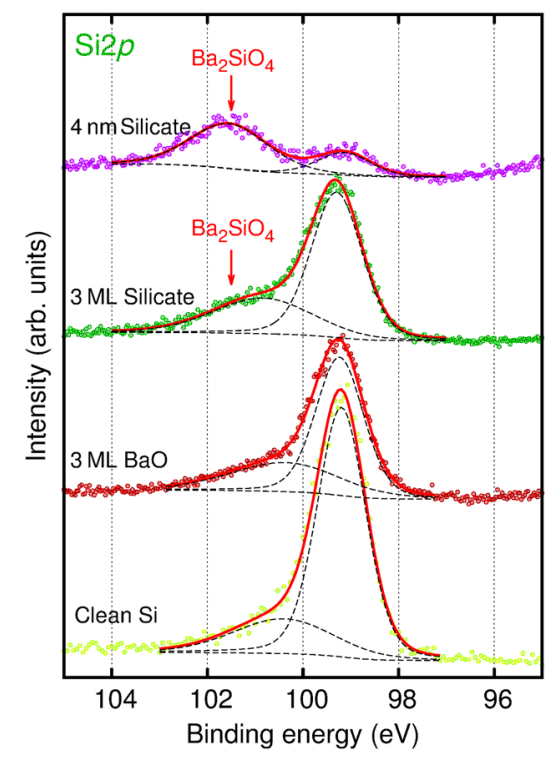

FIG. 1. XP spectra of the $\mathrm{Si} 2 p$ level during silicate formation. From bottom to top: Clean $\mathrm{Si}(100)$ surface, after deposition of $3 \mathrm{ML}$ of $\mathrm{BaO}$ grown at room temperature, after annealing this layer for $30 \mathrm{~min}$ at $650{ }^{\circ} \mathrm{C}$. Top: $4 \mathrm{~nm}$ of Ba silicate grown at a substrate temperature of $650{ }^{\circ} \mathrm{C}$.
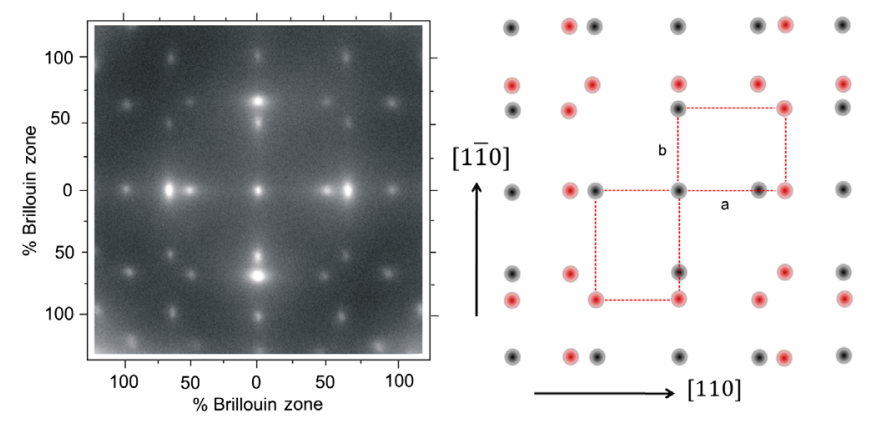

FIG. 2. Left: LEED image of a 2.5-nm-thick Ba-silicate structure at a primary energy of $70 \mathrm{eV}$. For clarification, a schematic of the LEED pattern is shown on the right. This schematic illustrates that the spots of the two domains of the $\mathrm{Si}$ $p(2 \times 1)$ (dark spots), in fact, partly coincide with those of the two rectangular unit cells of the $\mathrm{Ba}_{2} \mathrm{SiO}_{4}$ domains, which, however, produce additional spots (red).

film thickness is calibrated using TEM; see below. As seen there, two ordered rectangular crystalline domains can be discriminated, which are aligned along the $[110]$ and $[1 \overline{1} 0]$ directions of the $\operatorname{Si}(100)$ surface. The shorter axis in reciprocal space coincides within error margins with the $\times 2$ spots of the $(2 \times 1)$ reconstruction of the clean $\mathrm{Si}(100)$ surface, while the other one is longer by one-third. Precise evaluation and transformation to real space of these diffraction spots yields lattice constants of $|\vec{a}|=$ $5.77 \pm 0.05 \AA$ and $|\vec{b}|=7.61 \pm 0.05 \AA$. These values compare well with two axes of the orthorhombic bulk $\mathrm{Ba}_{2} \mathrm{SiO}_{4}$ crystal $(a=5.81 \AA, b=7.51 \AA, c=10.21 \AA)$ [37]. This demonstrates strong preference for epitaxial growth with the $a$ and $b$ axes aligned along the [110] and [110] directions of $\mathrm{Si}(100)$, respectively, and with the $c$ axis normal to the interface. Crystalline growth and similar values of lattice parameters $(a=5.77 \AA, b=7.44 \AA$, $c=10.20 \AA$ ) for Ba silicate are also reported in a previous combined RHEED and x-ray diffraction study on Sr- and Ba-silicate films grown at comparable temperatures [35]. While the lattice constant $a$ agrees with the bulk lattice constant within error bars, the lattice constant $b$ deviates from the bulk value in opposite direction to our result, possibly because growth of Ba silicate in Ref. [35] was started on a $\mathrm{SiSr}_{2}$-terminated $\mathrm{Si}$ surface, which may result in the admixture of $\mathrm{Sr}$ for very thin layers.

Slight deviations from the exact stoichiometry within the films are to be expected, since during silicate formation the $\mathrm{Si}$ species has to diffuse into and through the silicate films already present [32]. For this process to be effective, a finite gradient of Si concentration within the film is required, and, thus, a deviation from exact stoichiometry. This finding is in agreement with the observation of reduced crystalline quality with increasing film thickness (see, also, below).

In order to get more insight into the details of the growth mechanism and into the interface properties on the atomic scale, we perform cross-sectional TEM investigations. The 


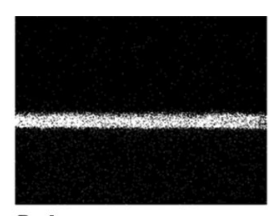

$\mathrm{Ba} L_{\alpha_{1}}$
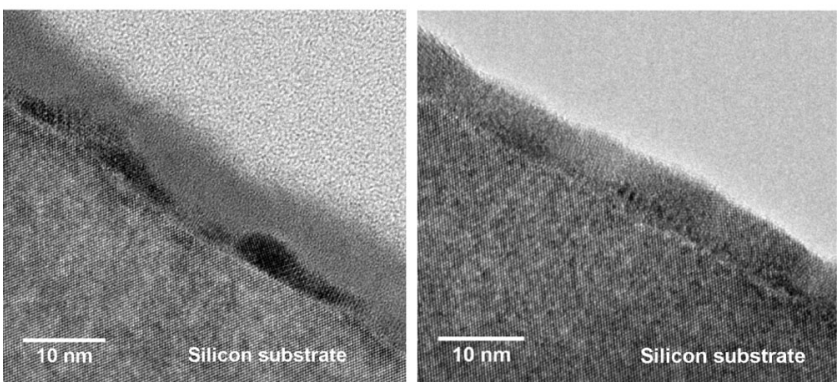

FIG. 3. Top: Cross-sectional EDX microspectroscopy using the $L \alpha$ emission of $\mathrm{Ba}$ (left) and the $K \alpha$ emissions of $\mathrm{Si}$ and oxygen. Bottom: Cross-sectional views in TEM of the Si-Ba-silicate interface. The Si wafer is oriented along [110]. Left: $10 \mathrm{~nm}$ of silicate. Here, only about half of the layer thickness is crystalline with different crystal orientations of the silicate. Right: $5 \mathrm{~nm}$ of silicate. Here, at many locations the whole silicate layer is crystalline. For more details, see Fig. 4.

TEM results are shown in Figs. 3 and 4. We start with an elemental analysis carried out with scanning TEM and EDX analysis at the $K_{\alpha 1}$ edges of oxygen and $\mathrm{Si}$, and the $L_{\alpha 1}$ edge of $\mathrm{Ba}$, respectively, for a nominally 10 -nm-thick silicate film. Within the resolution of this method of about $2 \mathrm{~nm}$, we can directly observe the homogeneous concentrations of $\mathrm{Ba}$ and oxygen within the layer, while the $\mathrm{Si}$ signal fades out at the edge to the epoxy layer, as expected from the arguments given above.

As a consequence of this concentration gradient of $\mathrm{Si}$, the 10 -nm-thick film is only crystalline close to the Si-silicate
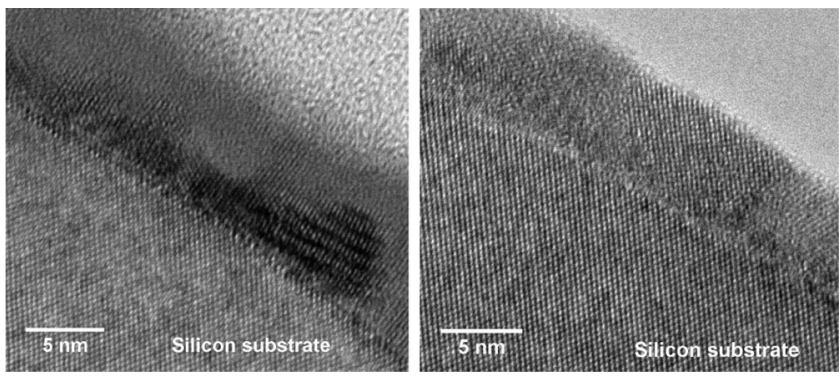

FIG. 4. Enlarged view of the characteristic Si-silicate interface for 10-nm- (left) and 5-nm-thick silicate layers. The interface contains some roughness on the atomic scale, and the silicate is crystalline at the interface. Several azimuthal orientations can be identified. In the left picture, the crystalline structure above the $\mathrm{Si}$ substrate can be identified mostly on the shortest silicate axis (5.80 $\AA$ ) parallel to the $[110]$ interface direction of Si. It is partly modulated with a $-\mathrm{nm}$ periodicity normal to the interface corresponding to the $c$ axis of the silicate (10.22 $\AA$ ). Right picture: The center part shows crystallites with the $b$ axis $(7.45 \AA) \|[110]$. interface but lacks crystallinity in about half of the thickness, as seen in the lower left part of Fig. 3. In accordance with the LEED results shown above, the main crystallographic orientations of Ba-silicate are along the [110] directions of $\mathrm{Si}$ and crystallites of both types of orientations, $[100]_{\mathrm{Ba}_{2} \mathrm{SO}_{4}} \|[110]_{\mathrm{Si}(100)}$, and $[010]_{\mathrm{Ba}_{2} \mathrm{SO}_{4}} \|[110]_{\mathrm{Si}(100)}$ (darker sections) can be identified. At some locations, also an intensity modulation normal to the interface with a period of $1.0 \mathrm{~nm}$ can be observed, which corresponds to lattice constant $c$ of the crystallites.

The average lateral extension of the crystallites is about $15 \mathrm{~nm}$, which agrees well with that deduced from the spot profile widths in LEED. If the thickness of the Ba-silicate film is reduced to $5 \mathrm{~nm}$, as shown on the bottom right of Fig. 3, the overall crystallinity of the film is improved considerably, since crystalline regions now extend up to the full layer thickness. Apart from extended crystallites with the same orientation and also a similar average size as just described for the 10-nm film, a minority of much smaller nanocrystallites with other orientations is visible. This species may even still be amorphous, since no signal above the diffuse background is detectable with LEED. The relative amount of crystalline and amorphous silicate will depend on the detailed growth conditions, which, however, are not varied systematically enough in this study to draw any quantitative conclusions.

These findings show that no silicide, metallic, or oxidic monolayers are necessary for crystalline growth of $\mathrm{Ba}_{2} \mathrm{SiO}_{4}$ on $\mathrm{Si}(100)$. The reaction to silicate is clearly activated, since close to room temperature a preference for oxide formation exists, when $\mathrm{Ba}$ or $\mathrm{Sr}$ are evaporated in oxygen atmosphere [27,28,35,36]. This activation must be mainly necessary for the formation of $\mathrm{SiO}_{4}^{4-}$ tetraeders, which are linked by the $\mathrm{Ba}$ ions in between. Since $\mathrm{SiO}_{4}^{4-}$ is formed only in presence of $\mathrm{Ba}$ or $\mathrm{Sr}$, a crystalline layer must already be formed within the first silicate layer. This short-range interaction and the good fit to the $\mathrm{Si}$ substrate lattice favors the orientation of the lattice constants $a$ and $b$ along the Si close-packed directions, whereas lattice constant $\mathrm{c}$ is determined only by layer stacking. On the other hand, local silicate formation reduces coupling to the substrate sufficiently in order to make diffusion sufficiently fast for perfect alignment and further crystalline growth.

Since $\mathrm{Ba}$ silicate is formed under reactive growth conditions here, with $\mathrm{Si}$ diffusion being the most crucial step, it is not surprising that the interface between $\mathrm{Si}$ and silicate is rough, also on the atomic scale, as demonstrated in Fig. 4 for 5- and 10-nm-thick silicate films. Nevertheless, as judged from XPS, there are no signs of formation of other chemical species, neither in XPS nor in TEM. An enhanced concentration of structural defects at the interface has consequences for the electrical properties (see below), since they may also be coupled with local compositional fluctuations on the atomic scale. 


\section{Characterization of electronic properties}

In the following, we concentrate on 6.25-nm-thick Ba-silicate layers. In order to determine the width of the band gap, we perform EELS measurements on these samples using a standard electron gun as the electron source, yielding an energy resolution of about $0.5 \mathrm{eV}$. As seen from the typical EELS spectrum shown in Fig. 5, these silicate films have a clear band gap with a small excitation background of about $10^{-2}$ of the elastically scattered peak intensity. A gradual increase is seen for loss energies above $4 \mathrm{eV}$, which we ascribe to the excitation of Frenkel excitons. Since the series of excitations cannot be resolved, only single peaks is used in the fit for the excitons, together with further Gaussian peaks for bulk silicate excitations. The steep increase close to 7-eV loss energy indicates the dominance of bulk excitations at higher loss energies. An estimate for the bulk band gap of Ba silicate is obtained from the linear extrapolation of this steep increase back to zero loss intensity to be $5.7 \pm 0.2 \mathrm{eV}$. This value coincides with the first abrupt change of slope of the loss intensity, which can be taken as a further indication of the onset of bulk excitations. It also agrees within error bars with that found for annealed mixed $\mathrm{SrBa}$-oxide layers grown at room temperature [32].

With this information, we now determine the band offsets and the band alignment of $\mathrm{Ba}$ silicate with respect to $p$-doped $\mathrm{Si}(100)$ by XPS using the method of Waldrop et al. [38]. The method is based on a comparison of the energetic distance of arbitrary core lines from the valenceband edges of bulk materials of the silicon substrate and of the insulator adsorbate, with the energetic distance of the same core lines but now for the silicon or insulator heterojunction. Core levels of $\mathrm{Si} 2 p, \mathrm{Ba} 3 d$, and $\mathrm{O} 1 s$ are used. Instead of bulk material for the silicate, we use the values for the 6.25-nm-thick film and compare it with the 3-ML-thick silicate film. More details of the data evaluation are described in Ref. [28]. The result shown in Fig. 6 reveals fairly symmetric band offsets of valence and

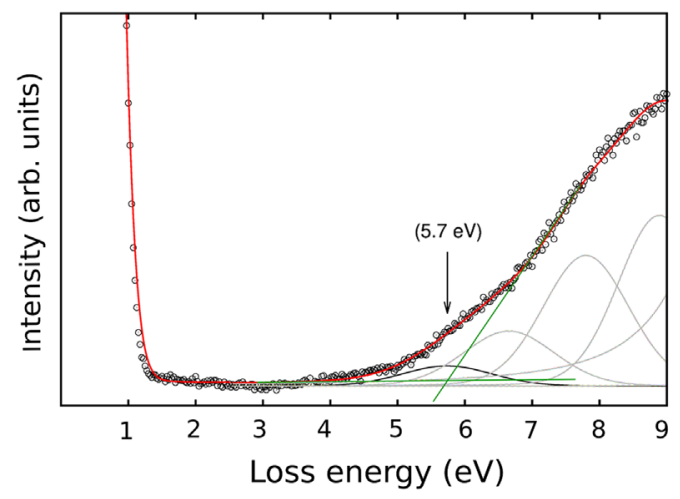

FIG. 5. Electron-energy-loss spectrum on a 6.25-nm-thick Ba-silicate layer. The band gap is determined by linear extrapolation of the loss intensity on the right side, not considering the excitonic loss (black curve).

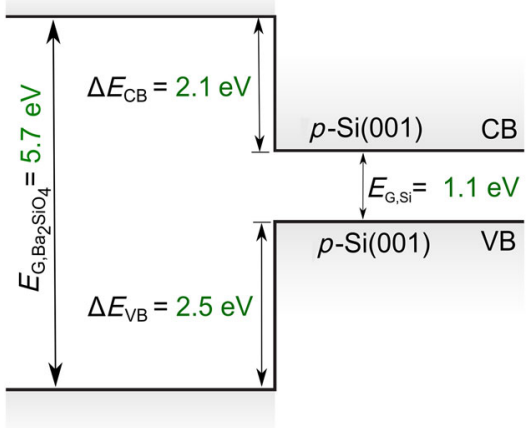

FIG. 6. Band offset as determined with XPS between $p$-Si and $\mathrm{Ba}_{2} \mathrm{SiO}_{4}$.

conduction bands, indicating a small concentration of polarization charges at the interface between $\mathrm{Si}$ and silicate. This finding is in agreement with the small core-level shift observed in Fig. 1 of the Si $2 p$ emission upon formation of silicate. The bulk Si peak is shifted by less than $0.1 \mathrm{eV}$, which is within the error bar of our determination of band alignments.

MOS diodes are fabricated completely in UHV with oxide thicknesses between 6.25 and $13 \mathrm{~nm}$ grown at a sample temperature of $650^{\circ} \mathrm{C}$ under a constant flux of $\mathrm{Ba}$ in oxygen background pressure. These layers are capped with a monolayer of $\mathrm{Al}$ and $150 \mathrm{~nm}$ of $\mathrm{Au}$, as mentioned above. These samples turn out to be stable in air so that $C V$ and $I-V$ measurements can be carried ex situ. A typical $C V$ curve is shown in the left part of Fig. 7. As seen there, hysteresis turns out to be below $1 \mathrm{mV}$ at the steepest slope, which on the scale shown in this figure, is not visible. This demonstrates that the density of rechargeable traps near the interface is extremely low. On the other hand, a large positive flatband voltage of $+0.8 \mathrm{~V}$ is measured as compared to an estimated value close to zero (between +0.07 and $-0.13 \mathrm{~V}$ ) derived from the Si doping, electron affinity, and the Au work function. This shift of flat band voltage is an indication of the presence of dipole (polarization) charges either at the $\mathrm{Si}$ silicate or at the silicate-gold interface or of trapped negative charges within the insulator. The majority of charges, however, cannot be located at the Si-silicate interface: As seen from Fig. 6, the band
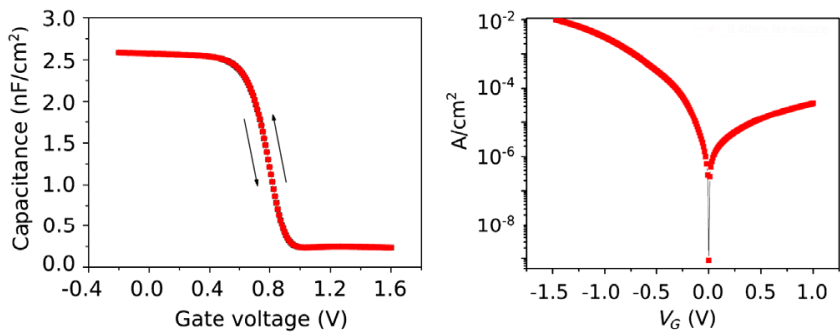

FIG. 7. Left: $C V$ curve measured for a 6.25-nm-thick $\mathrm{Ba}_{2} \mathrm{SiO}_{4}$ layer on $\mathrm{Si}(001)$. Right: $I-V$ curve for a 9.4-nm-thick Ba-silicate film. 
alignment at this interface is close to the value expected when no charge transfer occurs when the Si-silicate interface is formed. The Fermi level of $p$-doped $\mathrm{Si}$ at room temperature and at the given doping concentration is close to $0.24 \mathrm{eV}$ above the valence-band edge. Assuming no charge transfer and midgap location of $E_{F}$ within the insulator, band offsets for the valence bands of $2.61 \mathrm{eV}$ and of 2.09 for the conduction bands result. This assumption agrees with the small shift of the $\mathrm{Si} 2 p$ peak during formation of the first few Ba-silicate layers of $0.1 \mathrm{eV}$ (see Fig. 1). When growing thicker layers, this shift again remains within error bars of $\pm 0.05 \mathrm{eV}$ so that the accumulation of charges within the silicate layers, at least during growth, can be excluded. Therefore, it is likely that dipole formation at the silicate-gold interface and here, in particular, at the $\mathrm{Al}$ interface layer, is responsible for the observed shift. Interestingly, the polarity of the corresponding interface dipole that has been observed recently for mixed BaSr oxide layers [33] and has also been predicted theoretically [39] is reversed compared to the oxide layers. However, no systematic study of Al layer thickness is carried our here to explore its origin in more detail.

The leakage current through the silicate is measured taking $I-V$ curves. An $I-V$ curve at dc for a 9.4-nm-thick film is shown in the right part of Fig. 7, yielding a leakage current of $3 \mathrm{~mA} / \mathrm{cm}^{2}$ at $-1 \mathrm{~V}$. The equivalent oxide thickness [capacitance-equivalent thickness (CET)] for this layer is $1.6 \mathrm{~nm}$ (see below) and, thus, still fulfills the critical limits of leakage currents for practical applications [16].

From frequency-dependent small-signal admittance measurements, we determine quantitatively the capacitances in strong accumulation using a frequency-dependent three-element model in order to account for leakage currents and series resistances. The series resistance is separately measured on a sample with only back contacts. The CET to $\mathrm{SiO}_{2}$ is calculated from the capacitance value measured in the accumulation region without considering the quantum-mechanical effects. Here, all the electrical measurements are taken with the same size of windows

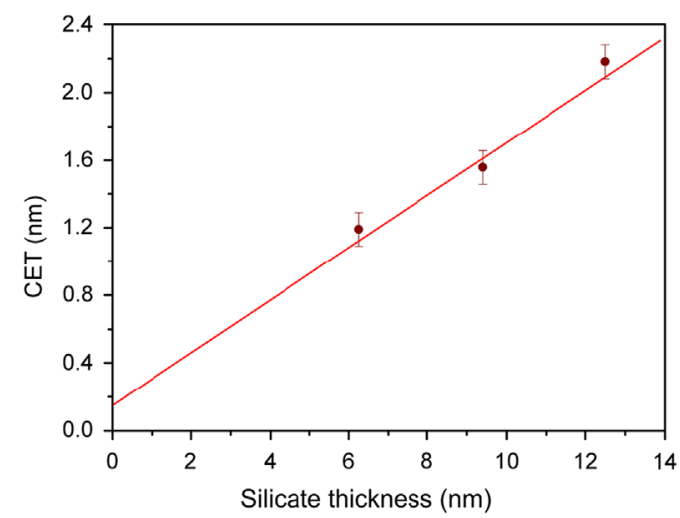

FIG. 8. CET as obtained by variation of silicate thickness. The nonzero intersection at zero thickness corresponds to the $\mathrm{Si}$ accumulation capacitance. (area of $4 \times 10^{-4} \mathrm{~cm}^{2}$ ). The gate metal or field oxide overlap capacitances are carefully subtracted. We plot in Fig. 8 the averages of the CET values for each silicate layer thickness tested. The error bars are determined from the scatter of the values for all probe windows. Within these error limits, the data fall on a straight line. From the slope, a relative dielectric constant of $\epsilon=22.8 \pm 0.2$ is determined. Taking the empirical correlation between the band gap and the value of $\epsilon$ [1], our values obtained here support this correlation fully. Since silicate layers with a thickness down to at least $3 \mathrm{~nm}$ can be produced without problems, CETs of $0.5 \mathrm{~nm}$ and less are feasible with this material. Also expected is the positive intercept with the CET axis at $0.18 \mathrm{~nm}$ due to the finite semiconductor capacitance in strong accumulation that has not been considered so far.

Frequency-dependent measurements are also used to determine the density of interface traps $D_{\mathrm{it}}$ under flatband conditions $(-0.32 \mathrm{eV}$ below midgap at the doping level of our samples) using the conductance method [40]. $D_{\mathrm{it}}$ is determined from the peak values of conductance $G_{\text {it }} / \omega$ and the width in the frequency domain. From these curves and the known area of our windows, we calculate values for the interface trap density $D_{\text {it }}$ to be around $1 \pm 0.5 \times 10^{13} \mathrm{eV}^{-1} \mathrm{~cm}^{-2}$. While these values scatter between samples, there is no clear tendency as a function of silicate thickness. At midgap, they may be a factor of 3 to 5 lower.

There are several possible reasons for these high values of $D_{\text {it }}$. Two of them are, as already mentioned, the need for reactive formation of the $\mathrm{Ba}$ silicate and the diffusive transport of $\mathrm{Si}$ atoms into the silicate and to the silicate surface for further silicate formation. Especially, the latter process causes a rough interface on the atomic scale between $\mathrm{Si}$ and the silicate, as seen in Figs. 3 and 4. Furthermore, the lattices of $\mathrm{Si}$ and silicate have quite different symmetries (cubic vs tetragonal) and imperfect recrystallization, particularly, at the interface, can be a source for local defects. The roughness may cause local strain fields that are efficient in electron scattering. This finding underlines again the importance of control of the interface properties on the atomic scale, which is difficult by direct means of spectroscopy and microscopy. However, indirect methods like the variation of the growth modes may give access.

\section{SUMMARY AND CONCLUSIONS}

We characterize in this study the formation of crystalline $\mathrm{Ba}_{2} \mathrm{SiO}_{4}$ on $\mathrm{Si}(100)$ using a combination of diffraction, microscopy, electron spectroscopy, and frequencydependent electrical measurements. To our surprise, crystalline growth at a surface temperature of $650^{\circ} \mathrm{C}$ works on the bare $\mathrm{Si}$ surface; i.e., it does not need any surface passivation or interface layer because of self-passivation. Lattice match to the substrate, at least for one crystal axis, however, still is important for epitaxial and crystalline growth, since with Sr silicate, which has the same crystal 
structure and stoichiometry but lattice constants that are about 4\% smaller than in Ba silicate [37], no crystalline growth is achieved so far, in agreement with Ref. [36].

Because of reactive growth, which requires $\mathrm{Si}$ atoms to diffuse through existing silicate layers, thicknessdependent deviations from exact stoichiometry are expected. This concentration gradient is most likely the reason for only partial crystallinity for layers above $6 \mathrm{~nm}$ thickness (under our growth conditions). The local deficiency or surplus of $\mathrm{Si}$ atoms causes variable concentrations of defects, which may be a source for leakage currents through the dielectric. This gradient is not larger than $0.5 \%$ per $\mathrm{nm}$, as estimated from preliminary secondary ion mass spectrometry (SIMS) measurements.

Taking into account its high thermal stability (desorption from the Si surface above $750{ }^{\circ} \mathrm{C}$ under UHV conditions) and the relatively small tendency for uptake of crystal water, the material with a relative dielectric constant of $\epsilon=22.8$ seems to be well suited as high- $k$ dielectric. Interestingly, and despite the obvious deficiency of $\mathrm{Si}$ in thicker layers, it has low concentrations of rechargeable bulk traps as well as acceptable ranges of leakage currents. The still high concentration of interface traps is most likely due to the relatively rough interface generated under the present growth conditions and indicates that better control of the interface properties is needed.

Summarizing, our investigations of the structural, chemical, and electrical properties of crystalline ultrathin Ba-silicate films on $\mathrm{Si}(100)$ and their interface open the route to a new class of insulating material that is free of oxide at the interface. While the material fulfills many requirements that are technically interesting (dielectric constant, thermal stability, band alignment), this material may greatly facilitate the manufacturing process due to the lack of an interface oxide. On the other hand, crystallinity of the films allows detailed control of interface properties on the atomic scale that are important for gate dielectrics and are not easily achievable with other materials.

\section{ACKNOWLEDGMENTS}

We gratefully acknowledge funding of this work by a fellowship from the Deutsche Akademische Austauschdienst (S. I.) and SIMS measurements by the group of U. Starke (MPI Stuttgart, Germany), as well as assistance in TEM investigations by F. Steinbach.

[1] J. Robertson and R. M. Wallace, High- $\kappa$ materials and metal gates for CMOS applications, Mater. Sci. Eng. R 88, 1 (2015).

[2] T. Ando, Ultimate scaling of high- $\kappa$ gate dielectrics: Higher- $\kappa$ or interfacial layer scavenging, Materials 5, 478 (2012).

[3] M. Copel, E. Cartier, V. Narayanan, M. C. Reuter, S. Guha, and N. Bojarczuk, Characterization of silicate/Si(001) interfaces, Appl. Phys. Lett. 81, 4227 (2002).
[4] M. Copel, M. C. Reuter, and P. Jamison, Interlayer composition of $\mathrm{HfO}_{2} / \mathrm{Si}(001)$ films, Appl. Phys. Lett. 85, 458 (2004).

[5] J.-P. Maria, D. Wicaksana, A. I. Kingon, B. Busch, H. Schulte, E. Garfunkel, and T. Gustafsson, High temperature stability in lanthanum and zirconia-based gate dielectrics, J. Appl. Phys. 90, 3476 (2001).

[6] H. Kim and P. C. McIntyre, Spinodal decomposition in amorphous metal-silicate thin films: Phase diagram analysis and interface effects on kinetics, J. Appl. Phys. 92, 5094 (2002).

[7] S. Stemmer, Z. Chen, C. G. Levi, P. S. Lysaght, B. Foran, J. A. Gisby, and J.R. Taylor, Application of metastable phase diagrams to silicate thin films for alternative gate dielectrics, Jpn. J. Appl. Phys. 42, 3593 (2003).

[8] J. Veres, S. Ogier, G. Lloyd, and D. de Leeuw, Gate insulators in organic field-effect transistors, Chem. Mater. 16, 4543 (2004).

[9] You Zhou and Shriram Ramanathan, Correlated electron materials and field effect transistors for logic: A review, Crit. Rev. Solid State Mater. Sci. 38, 286 (2013).

[10] Manuel Bibes, Javier E. Villegas, and Agnes Barthelemy, Ultrathin oxide films and interfaces for electronics and spintronics, Adv. Phys. 60, 5 (2011).

[11] J. Bass, CPP magnetoresistance of magnetic multilayers: A critical review, J. Magn. Magn. Mater. 408, 244 (2016).

[12] Vu Thanh Tra, Jan-Chi Yang, Ying-Hui Hsieh, Jiunn-Yuan Lin, Yi-Chun Chen, and Ying-Hao Chu, Controllable electrical conduction at complex oxide interfaces, Phys. Status Solidi RRL 8, 478 (2014).

[13] A. D. Caviglia, S. Gariglio, N. Reyren, D. Jaccard, T. Schneider, M. Gabay, S. Thiel, G. Hammerl, J. Mannhart, and J.-M. Triscone, Electric field control of the $\mathrm{LaAlO}_{3} / \mathrm{SrTiO}_{3}$ interface ground state, Nature (London) 456, 624 (2008).

[14] R. A. McKee, F. J. Walker, and M. F. Chisholm, Crystalline Oxides on Silicon: The First Five Monolayers, Phys. Rev. Lett. 81, 3014 (1998).

[15] D. G. Schlom and J. H. Haeni, A thermodynamic approach to selecting alternative gate dielectrics, MRS Bull. 27, 198 (2002).

[16] J. Robertson, High dielectric constant gate oxides for metal oxide Si transistors, Rep. Prog. Phys. 69, 327 (2006).

[17] J. H. Choi, Y. Mao, and J.P. Chang, Development of hafnium based high- $\kappa$ materials-A review, Mater. Sci. Eng. R 72, 97 (2011).

[18] Gang He, Zhaoqi Sun, Guang Li, and Lide Zhang, Review and perspective of Hf-based high- $\kappa$ gate dielectrics on silicon, Crit. Rev. Solid State Mater. Sci. 37, 131 (2012).

[19] Debashis Panda and Tseung-Yuen Tseng, Growth, dielectric properties, and memory device applications of $\mathrm{ZrO}_{2}$ thin films, Thin Solid Films 531, 1 (2013).

[20] L. M. R. Scolfaro, H. W. Leite Alves, P. D. Borges, J. C. Garcia, and E.F. da Silva, Jr., Structural, electronic, vibrational and dielectric properties of selected high- $\kappa$ semiconductor oxides, J. Phys. D 47413001 (2014).

[21] R. A. McKee, F. J. Walker, J. R. Conner, and E. D. Specht, Molecular beam epitaxy growth of epitaxial barium silicide, barium oxide, and barium titanate on silicon, Appl. Phys. Lett. 59, 782 (1991). 
[22] X. Hu, Y. Liang H. Li, Y. Yu, D. Marshall, Jr., J. Edwards, R. Droopad, X. Zhang, A. A. Demkov, and K. Moore, The interface of epitaxial $\mathrm{SrTiO}_{3}$ on silicon: In situ and ex situ studies, Appl. Phys. Lett. 82, 203 (2003).

[23] N. K. Bhuiyan, A. Matsuda, T. Yasumura, T. Tambo, and C. Tatsuyama, Study of epitaxial $\mathrm{SrTiO}_{3}$ (STO) thin films grown on $\mathrm{Si}(001)-(2 \times 1)$ substrates by molecular beam epitaxy, Appl. Surf. Sci. 216, 590 (2003).

[24] W. F. Xiang, H. B. Lu, Z. H. Chen, X. B. Lu, M. He, H. Tian, Y. L. Zhou, C. R. Li, and X. L. Ma, Heteroepitaxial growth of $\mathrm{LaAlO}_{3}$ films on $\mathrm{Si}(100)$ by laser molecular beam epitaxy, J. Cryst. Growth 271, 165 (2004).

[25] C. Merckling, G. Delhaye, M. El-Kazzi, S. Gaillard, Y. Rozier, L. Rapenne, B. Chenevier, O. Marty, G. SaintGirons, M. Gendry, Y. Robach, and G. Hollinger, Epitaxial growth of $\mathrm{LaAlO}_{3}$ on $\mathrm{Si}(001)$ using interface engineering, Microelectron. Reliab. 47, 540 (2007).

[26] R. A. McKee, F. J. Walker, and M. F. Chisholm, Physical structure and inversion charge at a semiconductor interface with a crystalline oxide, Science 293, 468 (2001).

[27] Y. Segal, J. W. Reiner, A. M. Kolpak, Z. Zhang, S. IsmailBeigi, C. H. Ahn, and F. J. Walker, Atomic Structure of the Epitaxial $\mathrm{BaO} / \mathrm{Si}(001)$ Interface, Phys. Rev. Lett. 102, 116101 (2009).

[28] J. Zachariae and H. Pfnür, Growth conditions, stoichiometry, and electronic structure of lattice-matched $\mathrm{SrO} / \mathrm{BaO}$ mixtures on Si(100), Phys. Rev. B 72, 075410 (2005).

[29] J. Zachariae and H. Pfnür, Surface morphology of epitaxial lattice-matched $\mathrm{BaSrO}$ on $\mathrm{Si}(001)$ and vicinal $\mathrm{Si}(001)-4^{\circ}$ [110] substrates, Surf. Sci. 600, 2785 (2006).

[30] D. Müller-Sajak, A. Cosceev, C. Brand, K. R. Hofmann, and H. Pfnür, Preparation and electrical characterization of amorphous $\mathrm{BaO}, \mathrm{SrO}$ and $\mathrm{Ba}_{0.7} \mathrm{Sr}_{0.3} \mathrm{O}$ as high- $\kappa$ gate dielectrics, Phys. Status Solidi C 7, 316 (2010).
[31] A. Cosceev, D. Müller-Sajak, H. Pfnür, and K. R. Hofmann, Investigation of the electrical properties of the alkaline-earth oxides $\mathrm{BaO}, \mathrm{SrO}$ and $\mathrm{Ba}_{0.7} \mathrm{Sr}_{0.3} \mathrm{O}$ on $\mathrm{Si}(001)$ as alternative gate dielectrics, Thin Solid Films 518, S281 (2010).

[32] D. Müller-Sajak, S. Islam, H. Pfnür, and K. R. Hofmann, Temperature stability of ultra-thin mixed $\mathrm{BaSr}$-oxide layers and their transformation, Nanotechnology 23, 305202 (2012).

[33] S. Islam, D. Müller-Sajak, K. R. Hofmann, and H. Pfnür, Epitaxial thin films of $\mathrm{BaSrO}$ as gate dielectric, Microelectron. Eng. 109, 152 (2013).

[34] R. Barany, E. G. King, and S. S. Todd, Heats of formation of crystalline silicates of strontium and barium, J. Am. Chem. Soc. 79, 3639 (1957).

[35] D. P. Norton, C. Park, Y. E. Lee, and J. D. Budai, Strontium silicide termination and silicate epitaxy on $\mathrm{Si}(001)$, J. Vac. Sci. Technol. B 20, 257 (2002).

[36] M. ElKazzi, G. Delhaye, C. Merckling, E. Bergignat, Y. Robach, G. Grenet, and G. Hollinger, Epitaxial growth of $\mathrm{SrO}$ on $\mathrm{Si}(001)$ : Chemical and thermal stability, J. Vac. Sci. Technol. A 25, 1505 (2007).

[37] G. Pieper, W. Eysel, and T. Hahn, Solid solubility and polymorphism in the system $\mathrm{Sr}_{2} \mathrm{SiO}_{4}-\mathrm{Sr}_{2} \mathrm{GeO}_{4}-\mathrm{Ba}_{2} \mathrm{GeO}_{4}-\mathrm{Ba}_{2} \mathrm{SiO}_{4}$, J. Am. Ceram. Soc. 55, 619 (1972).

[38] J. R. Waldrop, R. W. Grant, S. P. Kowalczyk, and E. A. Kraut, Measurement of semiconductor heterojunction band discontinuities by x-ray photoemission spectroscopy, J. Vac. Sci. Technol. A 3, 835 (1985).

[39] L. Lin, J. Robertson, and S. J. Clark, Shifting Schottky barrier heights with ultra-thin dielectric layers, Microelectron. Eng. 88, 1461 (2011).

[40] J. R. Brews, Rapid interface parametrization using a single MOS conductance curve, Solid State Electron. 26, 711 (1983). 